\title{
Study of coordination and selectivity in circuit breakers
}

\author{
Helder da Silva Rodrigues ${ }^{1}$
}

\begin{abstract}
${ }^{1}$ Programa de Pós-graduação em Engenharia Elétrica (PPGE), Universidade Federal do Amazonas (UFAM) - Av. General Rodrigo Octavio Jordão Ramos, 1200. Bairro: Coroado I. Manaus - Amazonas - Brasil.
\end{abstract}

Email: eng.helder.hdr@hotmail.com

Received: January $03^{\text {th }}, 2018$.

Accepted: February $01^{\text {th }}, 2018$.

Published: March $30^{\text {th }}, 2018$.

Copyright (C2016 by authors and Institute of Technology Galileo of Amazon (ITEGAM).

This work is licensed under the Creative Commons Attribution International

License (CC BY 4.0).

http://creativecommons.org/licenses/by/4.0/ (c) (1) (3) (2) Opean Actes:

\begin{abstract}
This paper aims to study the coordination and selectivity of the electrical system of the substations of the terminal tip of the wood, consisting of defining the settings of the protection devices, which in the occurrence of a short circuit is only affected the region of protection action, isolating the smallest part of the electrical system, in the shortest possible time without loss of production and equipment malfunctions, with the aid of nominal data and damage curves of the electrical equipment can generate "time versus current" graphs provided by the software digs siprotec . In the test carried out on the protection devices the three-phase currents of I> or I> were taken into account for the phase-earth currents $\mathrm{I} 0>>$ or I0>. The results of the tests were satisfactory due to the efficiency of the software disg siprotec.
\end{abstract}

Keywords: Coordination and logical selectivity; Electrical Protection System; Short circuit; Table Anse; Multifunction relay.

\section{Estudo de coordenação e seletividade em disjuntores}

\section{RESUMO}

Este artigo tem por finalidade estudo de coordenação e seletividade do sistema elétrico das subestações da terminal ponta da madeira, consistindo em definir os ajustes dos dispositivos de proteção, que na ocorrência de um curto-circuito seja somente afetada a região de atuação da proteção, isolando a menor parte do sistema elétrico, no menor tempo possível sem que haja perca de produção e avarias nos equipamentos, com auxílio dos dados nominais e curvas de dano dos equipamentos elétricos pode-se gerar gráficos a "tempo versus corrente" fornecidas pelo software digs siprotec. No ensaio realizado nos dispositivos de proteção foram levadas em consideração as correntes trifásicas de I $>>$ ou I $>$ quanto as correntes fase-terra $\mathrm{I} 0>>$ ou $\mathrm{I} 0>$. Os resultados dos testes foram satisfatório devido à eficiência do software disg siprotec.

Palavras Chaves: Coordenação e Seletividade Lógica; Sistema Elétrico de Proteção; CurtoCircuito; Tabela Anse; Relé Multifunção.

\section{INTRODUÇÃO}

Em todos os projetos elétricos que estejam relacionados ao setor elétrico, faz-se necessário a realização de estudos detalhados sobre as possíveis falhas elétricas, sendo proveniente do ambiente, das circunstâncias ou ate mesmo da instabilidade na qualidade da energia elétrica, o trabalho apresentado tem como objetivo apresentar os resultados e ajustes do estudo de seletividade e coordenação das proteções recomendadas para implantação nos dispositivos de proteção do sistema elétrico das subestações SE-381K-A e SE-381K-B, para o Terminal Marítimo de Ponta da Madeira. Nesse documento são apresentados os critérios adotados, bem como considerações sobre as quais foram definidos os ajustes recomendados e determinar as ordens de graduação dos dispositivos de proteção, com a finalidade de que, quando da ocorrência de uma falta no sistema elétrico, sejam desligados deste o menor número possível de circuitos (sistema seletivo), mantendo o restante do sistema em operação. Essa falha por sua vez de acordo com o nível de curto-circuito pode gerar desequilíbrio na rede de transmissão ou distribuição, fazendo necessário que haja coordenação entre os dispositivos de proteção, a fim de garantir a integridade física dos equipamentos sem que haja desligamento indevido dos demais, isolando o curto o mais próximo do evento. 
Para que haja êxito na proteção dos equipamentos de potência, é necessário que os mesmos estejam nas zonas controladas, local onde a coordenação e seletividade de proteção poderão se comunicar através dos dispositivos de proteção (rele multifunção, contactores disjuntores transformadores entre outros dispositivos). Para que os estudos de coordenação sejam eficientes é necessário ter os seguintes critérios:

- $\quad$ Seletividade. Atuar diretamente no problema isolando as demais zonas sem que haja desligamento da planta.

- $\quad$ Rapidez. Deverá atuar no menor tempo possível para garantir a integridade das pessoas e equipamentos

- Segurança. Garantir que as partes defeituosas sejam desligadas com segurança.

No mercado atualmente existem inúmeros software para proteção, podemos assim citar o [1] que traz uma série de benefícios um deles podemos enfatizar o gráfico de "tempo versus corrente" em que é possível identificar os valores de tempo e corrente de um determinado dispositivo de proteção e sua curva característica que cada dispositivo possui, sendo que fusíveis e alguns disjuntores de proteção tem sua curva fixa, já rele multifunção tem um série de parâmetros que podem ser modificado de acordo com a necessidade da proteção [2].

Munidos de todas essas informações podemos definir os parâmetros nos rele de proteção, definir zonas controladas e realizar estudo de coordenação e seletividade em Centro de Controle de Motores (CCM).

\section{OBJETIVOS DO TRABALHO}

Este trabalho tem como objetivo apresentar os resultados e ajustes do estudo de seletividade e coordenação das proteções recomendadas para implantação nos dispositivos de proteção do sistema elétrico das subestações SE-381K-A E SE-381K-B, para o Terminal Marítimo de Ponta da Madeira. Nesse documento são apresentados os critérios adotados, bem como considerações sobre as quais foram definidos os ajustes recomendados e determinar as ordens de graduação dos dispositivos de proteção, com a finalidade de que, quando da ocorrência de uma falta no sistema elétrico, sejam desligados deste o menor número possível de circuitos (sistema seletivo), mantendo o restante do sistema em operação.

Assim, contempla-se ne estedudo a análise do sistema de proteção das subestações SE-381k-A e SE-381k-B, se limitando aos seus respectivos alimentadores. Serão avaliados os ajustes dos dispositivos de proteção a jusante, subestações secundárias existentes, e caso necessário serão feitas propostas de alterações.

Os ajustes foram elaborados de forma a coordenar com os relés a montante e jusante minimizando as alterações dos ajustes já calculados para SE-318K-01.

Realizar estudos de proteção em disjuntores de media e alta tensão de acordo com a [3] a serem aplicados em coordenação e seletividade (seletividade para disjuntores de Potência), para que a principal solução seja através do estudo com as característica e curva de proteção existente de cada dispositivo de proteção não havendo solução faz-se necessário troca do dispositivo.

\section{OBJETIVOS ESPECÍFICOS}

1. Apresentar equipamentos e dispositivos que estão envolvidos na coordenação e seletividade tais como disjuntores de média e alta tensão, rele multifunção, fusíveis, chaves seccionadoras, transformadores de potência, transformadores de corrente objetivo desse capitulo é fornecer informações detalhadas as características de proteção de cada dispositivo ao qual serão enfatizados nos capítulos seguintes.

2. Explorar anomalias na seletividade de proteção tomando com exemplos transformadores de $230 \mathrm{kv}$ e disjuntores, paralelismo entre transformadores de potência, curva de proteção instantânea e sobrecorrente de terra, função 27, parâmetros de goose.

3. Desenvolver melhorias na qualidade e eficiência da proteção em subestação de acordo com a norma IEC 61850.

\section{METODOLOGIA}

Ao realizar estudo de coordenação e seletividade foi constatado inúmeras problemáticas assim como área onde a curva de proteção necessitava de uma precisão maior usando com a filosofia o goose.

Munidos dessas informações utilizamos a ferramenta de qualidade PDCA também chamada de ciclo de Shewhart - e uma ferramenta de gestão que tem por objetivo promover a melhoria continua dos processos por meio de um circuito de quatro acoes : palnejar ( plan),fazer (do), checar (check) e agir. Ao utilizarmos tal ferramenta mapeamos todas as vulnerabilidades das proteções nas subestações do Terminal Ponta da Madeira fazendo com que todos os ajustes estivem na zona de proteção utilizando o software $\{1]$.

\section{DESENVOLVIMENTO DO PROJETO}

Para a verificação do desempenho da proteção, foram considerados os valores de curto-circuito trifásicos, calculados e apresentados no estudo de curto-circuito.

Para o valor de curto-circuito fase-terra, considerou-se que o sistema de $13,8 \mathrm{kV}$ possui o valor de corrente de falta máximo limitado em 400A.

Este estudo se limitou à análise e determinação dos ajustes de todos os alimentadores das subestações SE-381K-A/B, em função das maiores cargas de cada alimentador. Os ajustes das subestações secundárias deverão ser avaliados e alterados pelo TERMINAL PONTA DA MADEIRA no estudo específico. Todos os ajustes recomendados neste estudo atenderão às condições operacionais apresentadas no estudo de curto-circuito.

\section{IV.1 PROTEÇÃO DE TRANSFORMADORES}

Conforme se pode observar nos diagramas unifilares da instalação, os transformadores de potência têm seus enrolamentos primários e secundários protegidos por relés multifunções do tipo [4]. 


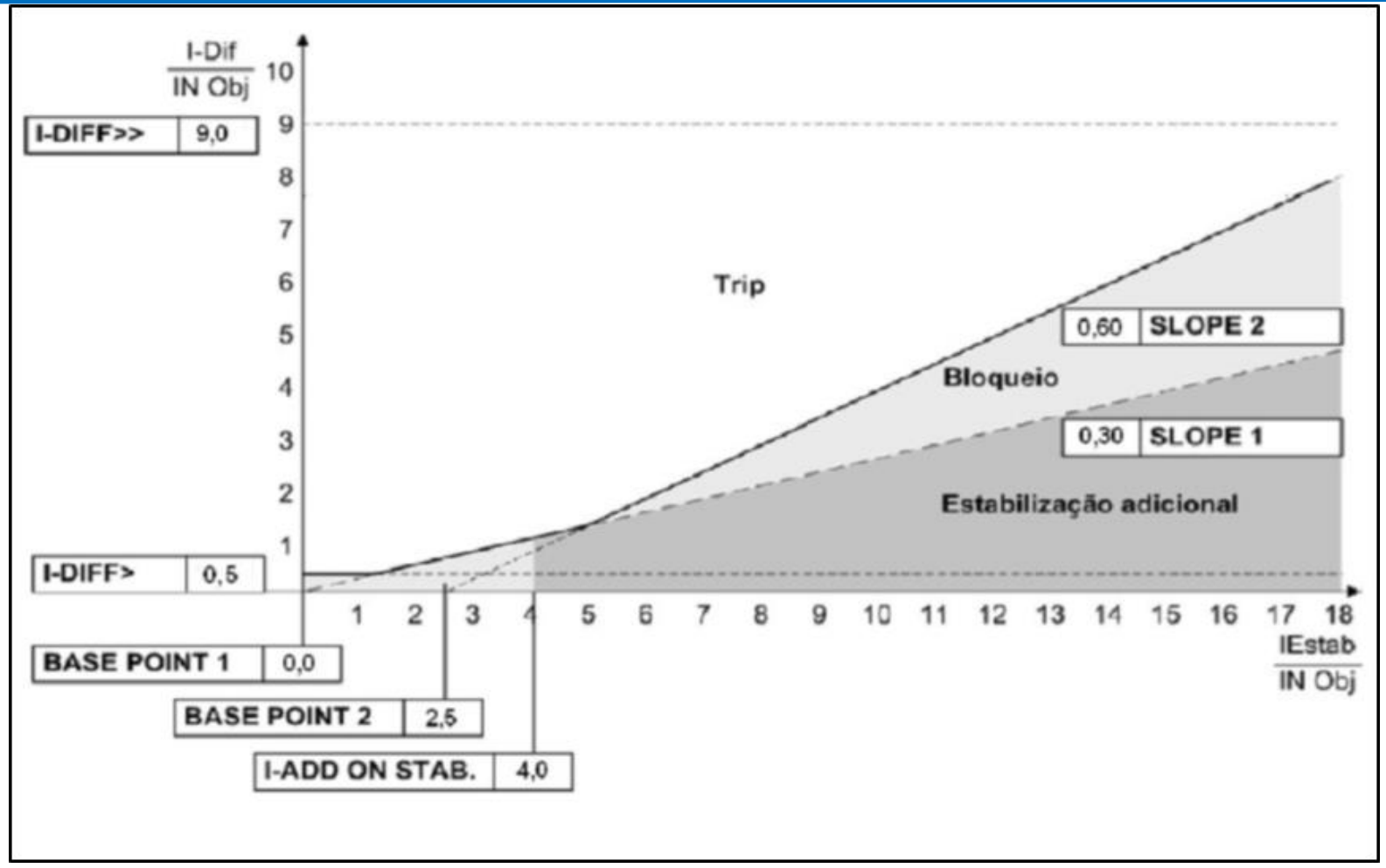

Figura 1: Proteção de transformadores.

Fonte: Autor, (2018).

\section{2 VERIFICAÇÃO CONFORME ANSI}

A curva do dispositivo de proteção deve atender aos requisitos da norma [6] para proteção quanto às altas correntes passantes pelo transformador.

Essas correntes podem ser oriundas de curto-circuito, mas também de partida de grandes motores.

A norma [6] define o tempo que um transformador deve suportar tensão plena em um dos enrolamentos com um determinado valor de corrente passante, sem danificação térmica ou mecânica do mesmo.

Nos coordenogramas desse estudo são apresentadas as curvas limites dos transformadores, em comparação com as curvas de operação dos dispositivos de proteção associados ao transformador. Para uma adequada proteção, a curva do dispositivo responsável pela proteção deve operar sempre em um tempo inferior ao limite do transformador [7][8].

\section{3 ESQUEMA TÍPICO DE SELETIVIDADE CONVENCIONAL}

A seletividade convencional (temporizada) executa a proteção de backup do sistema. Uma vez que existir falha na comunicação entre as IED's e não atuação de falha de disjuntor, a seletividade convencional atuará, isolando o circuito em falha do sistema.

Para a seletividade temporizada, assumiu-se uma não coordenação entre as interligações, uma vez que as mesmas protegem um mesmo elemento. A seguir tem-se ilustrado diversos casos de atuação da seletividade convencional.

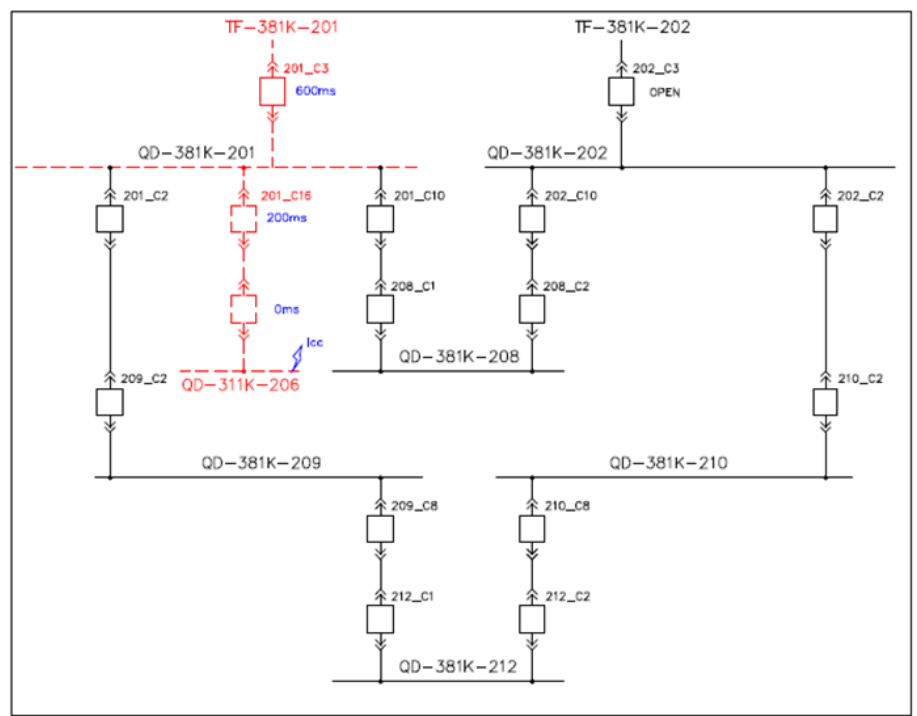

Figura 2: Esquema de seletividade convencional.

Fonte: Autor, (2018).

Para um curto no QD-311K-206 (SE-311K-D), considerando o TF-381K-202 fora de operação, o ramal alimentado pelo cubículo 201_C16 será sensibilizado. O relé da entrada do QD-311k-206 (SE-311K-D) atuará em 0ms, em caso de falha deste, o relé do cubículo 201_C16 atua em 200ms. Caso o relé do cubículo 201_C16 falhe, o relé do cubículo 201_C3 atua em 600ms [9].

\section{IV.4 ESQUEMA DE SELETIVIDADE APÓS MELHORIA DE ESTUDO DE PROTEÇÃO}

De modo a obter um sistema elétrico extremamente seguro, com seus dispositivos de proteção operando de forma bastante sensível e rápida, adotou-se neste projeto a 
implementação da seletividade lógica, para faltas trifásicas, no nível de $13,8 \mathrm{kV}$.

Para as faltas fase terra, devido ao sistema de aterramento ser através de resistores de baixo valor Ôhmico (400A-10seg), os tempos de seletividade lógica não serão aplicados.

A seletividade lógica consiste basicamente em uma rede de relés de proteção, interligados e ajustados em dois níveis de tempo diferenciados para cada dispositivo. Um dos níveis de tempo refere-se à coordenação da proteção de modo convencional, que opera em retaguarda. Já o outro nível possui tempos de operação bastante baixos, que dependem de uma série de bloqueios para que o sistema possa operar de forma seletiva.

Seu propósito é de que todos os relés em cascata possam ter suas unidades de proteção de sobrecorrente instantâneas habilitadas, sem que isso signifique perda de seletividade na atuação e eliminação da falta em um determinado circuito. $\mathrm{O}$ resultado é a diminuição do tempo de coordenação entre os relés e do tempo de eliminação da falta.

Esta função traduz-se no envio de um sinal discreto de um determinado relé sensibilizado por uma corrente de falta a um relé a montante, o qual também está sentindo uma falta suficiente para sensibilizar qualquer uma de suas unidades instantâneas. $\mathrm{O}$ relé a montante percebe através de uma entrada lógica, que o relé a jusante está sensibilizado para atuação, e bloqueará o "trip" de seletividade lógica instantânea.

A seletividade lógica poderá existir em diversos degraus. Assim, é necessário que o relé, ao sentir entrada de seletividade lógica fechada indicando que um relé a jusante está sentindo a falta, deverá bloquear somente a atuação de "trip" de seletividade lógica instantânea. Caso estas funções instantâneas também sejam sensibilizadas por correntes de valor maior que o seu "pick-up", a saída de seletividade lógica deverá fechar para bloquear o relé que está no próximo degrau de seletividade.

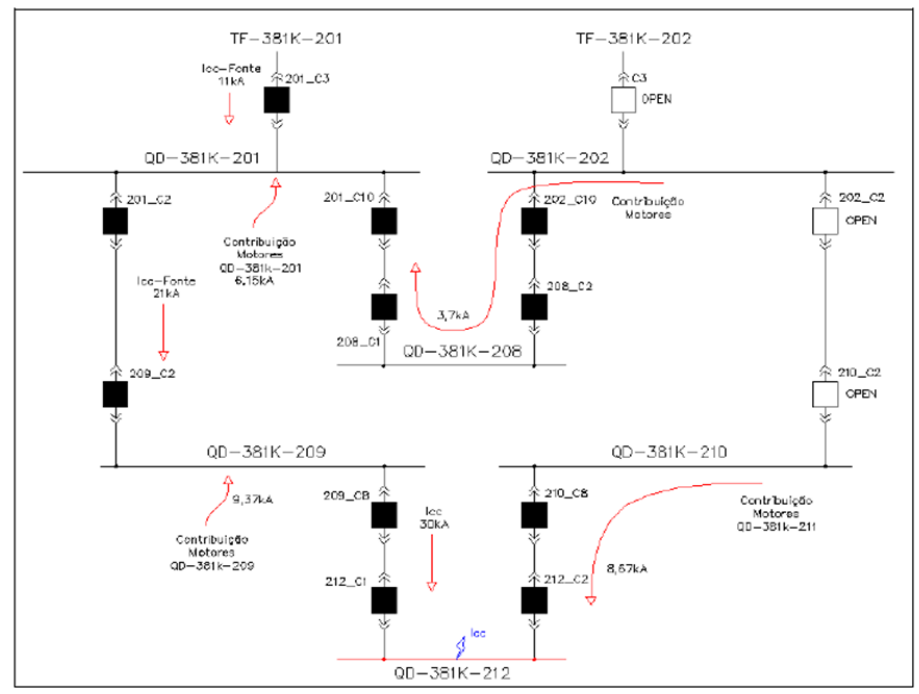

Figura 3: Esquema de seletividade convencional.

Fonte: Autor, (2018).

\section{RESULTADOS E GANHOS TANGIVEIS}

Em dezembro de 2015 a produção anual da companhia foi de 150 milhões em toneladas embarcadas porém essa quantidade vem aumentando gradativamente todos os anos com isso fez-se necessário revitalizações de várias subestações que estavam deterioradas, pois muitos dos dispositivos de proteção estavam obsoletos com isso a produção parava repentinamente pois tais dispositivos devido fadiga e corrosão do tempo na desenvolviam seu papel principal que era coordenar e proteger determinados equipamentos.

Foi realizado retrofit em $70 \%$ das subestações onde retiramos todos os dispositivos obsoletos e adquirindo novos com mais qualidade precisão e atualidade, com isso visando maior qualidade de energia e precisão do estudo de coordenação e seletividade podemos citar ganhos tangíveis.

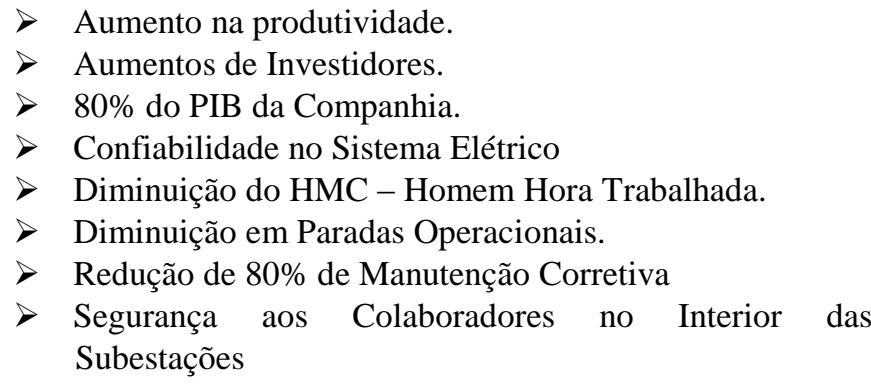

\section{V.1 GANHOS INTANGÍVEIS}

No estudo de coordenação e seletividade podemos ainda citar com grande orgulho e satisfação tais resultados obtidos ao decorrer de todo esse projeto pois não apenas trouxe benefício a companhia mas também toda a uma comunidade, fazendo com novos investimentos sejam aplicados trazendo bem em comum a todos.

$>$ Valorização dos empregados.

$>$ Qualidade de vida.

$>$ Remuneração sobre a produção da companhia.

$>$ Investimentos da comunidade

$>$ Abertura de novos portos de trabalho

\section{CONCLUSÕES E RECOMENDAÇÕES}

O sistema elétrico das subestações SE-381K-A E SE381K-B apresentará seletividade e proteção adequadas para os dispositivos de proteção em caso de ocorrência de curto-circuito e sobrecargas, considerando-se a implantação dos ajustes recomendados neste estudo.

Para as subestações secundárias existentes, com entrada seca, utilizou-se para a análise da proteção, o ajuste do dispositivo de proteção que alimenta a carga de maior potência.

Quando necessário, foi proposto alteração nos ajustes destes dispositivos de proteção com o objetivo de manter a coordenação com os dispositivos de proteção a montante, ou seja, relés alimentadores das subestações SE-381K-A e SE-381K-B. Os ajustes dos demais alimentadores das subestações secundárias existentes devem ser alterados em estudo específico.

O sistema de proteção foi projetado considerando as piores condições operacionais.

Entretanto recomenda-se que as cargas alimentadas a partir das subestações SE-381k-A e SE-381k-B, que possuem a flexibilidade de alimentação dupla a partir das chaves seccionadores instaladas nos painéis QD-381K-204 e QD-381K213, sejam distribuídas da forma mais uniforme possível. 
Isto irá diminuir as contribuições motóricas através dos alimentadores e manter o carregamento dos transformadores de 230 kV, das subestações SE-381k-A e SE-381k-B mais uniforme.

Estes ajustes são necessários para garantir a sensibilização para faltas trifásicas e bifásicas.

Os alimentadores, a partir dos painéis QD-381k-211/219, consistem em subestações que contem transformadores com potência da ordem de 10 MVA. Para coordenar as funções instantâneas das entradas e primários destes transformadores com os IEDs dos painéis QD- 381k-211/219 os ajustes deverão ser menores ou iguais a $4 \mathrm{kA}$. Devido à corrente de magnetização destes transformadores poderão ocorrer atuações indevidas. Para evitar esta atuação indevida deve-se ser habilitada, nos IEDs dos primários dos trafos, a função INRUSH RESTRAINT que terá a função de bloquear a função 50 quando da presença de $2^{\circ}$ harmônica, o que acontece quando de sua magnetização/energização. Portanto as novas subestações alimentadas diretamente, através do QD-381k-211/219 e que possuem esta função poderão ser alimentadas sem o paralelismo dos transformadores TF-381k-214/215.

Recomenda-se que seja desenvolvido um criterioso trabalho de comissionamento e testes das funções de seletividade lógica e falha de disjuntor. Para tanto, deve-se observar os bancos de dados dos ajustes da proteção, o descritivo de funcionamento da seletividade lógica e os diagramas funcionais dos painéis.

\section{REFERÊNCIAS}

[1] Arc Protection: Softtware SIPROTEC and DIGS: protection, automation devires support Arc Protection, Benetti from extremely fast tripp. SIEMENS, Infrastructure \& Cities Sector Smart Grid Division Energy Automation Humboldtstr. 5990459 Nuremberg, Germany 2012.

[2] SIEMENS. BETA Low voltage circuit protection characteristics curves for catalog ET B1. Disponível em: $<$ http://industry.siemens.com.br/buildingtecnologies/pt/produtosbaixa-tensão/produção-eletrica/Fusiveis-Documents-

Caracterisitcas.pdf>. Acesso em: 25 nov. 2012.

[3] IEEE Guide for the Selection of Monitoring for Circuit Breakers. IEEE Std C37.10.1-2000. 2001.

[4] IEC 61850-Communication Networks and Systems in Substations;

http://domino.iec.ch/webstore/webstore.nsf/searchview/?SearchVi ew $=\&$ Se

archOrder $=4 \&$ SearchWV=TRUE $\&$ SearchMax $=1000 \&$ Query $=61$ $850 \&$ su bmit $=\mathrm{OK}$.

\section{[5] SIPROTEC 7SJ62 Multifunction Protection Relay.} SIEMENS, 2011.

[6] C37.91-1985. IEEE Guide for Protective Relay Applications to Power Transformers Reaffirmed 6-12-1990. Superseded by IEEE Std C37.91-2000 (Revision of IEEE Std C37.91-1985).
[7] Mamede Filho, João. Proteção do sistema elétrico de potência. Edição 2011. Cidade Rio de Janeiro: Editora LTC, 2016.

[8] Kagan, Nelson. Introdução aos sistemas de distribuição de energia elétrica. $2{ }^{\circ}$ Edição Revisada. Cidade São Paulo: Editora Blucher, 2010.

[9] Simone, Gilio Aluisio. Transformadores. $1{ }^{\circ}$ Edição. Cidade São Paulo: Editora SARAIVA, 2010. 